\title{
HEMORRAGIA SUBARACNOIDEEA COM TOMOGRAFIA DE CRÂNIO SEM SINAIS DE SANGRAMENTO
}

\author{
SILVIO FRANCISCO*, JOÃO BAPTISTA DOS REIS-FILHO**, AFONSO CARLOS NEVES**
}

\begin{abstract}
RESUMO - Foram observados 23 casos de pacientes com suspeita clínica de hemorragia subaracnoídea (HSA), com tomografia de crânio (CT) sem sinais de sangramento, com diagnóstico definitivo realizado por exame de líquido cefalorraquiano(LCR). Desses, 20 casos foram submetidos a angiografia cerebral. Estabeleceu-se a classificação clínica de Hunt \& Hess. Os principais achados neste estudo foram:1) um terço dos pacientes apresentou intervalo de tempo entre o ictus e a realização da CT e do LCR entre 24 e 48 horas, período de maior sensiblidade da CT; 2 ) dos pacientes estudados com angiografia cerebral, 55\% tiveram o diagnóstico de aneurisma com predomínio no território de carótida e cerebral anterior; 3) o grau clínico dominante foi Hunt \& Hess I e II. Todos os achados encontrados demonstraram a necessidade da realização de LCR em suspeita de HSA sem sinais de sangramento na CT.
\end{abstract}

PALAVRAS-CHAVE: hemorragia subaracnoídea, líquido cefalorraquiano, tomografia computadorizada.

\section{Subarachnoidal hemorrhage with cranial tomography without bleeding signals}

ABSTRACT -We observed 23 pacients with clinical hypothesis of subarachnoidal hemorrhage (SAH) having cranial tomography (CT) without bleeding signals. The final diagnosis of SAH was made upon the cerebrospinal fluid (CSF) results. Twenty cases were submitted to brain angiography. They were focused under the clinical classification of Hunt \& Hess. The main results were: 1) 1/3 of patients had interval time between 24 and 48 hours after ictus and before CT and CSF making; this was the most sensitive CT time; 2) 55\% of patients with brain angiography had aneurysm predominantly in the carotid and anterior cerebral artery territory; 3) the dominant clinical degree was Hunt \& Hess I e II. Conclusion; the results demonstrate the need of CSF test in case of SAH clinical diagnosis with CT showing no bleeding signals.

KEY WORDS: subarachnoidal hemorrhage, cerebrospinal fluid, computerized tomography.

Hemorragia subaracnoidea (HSA) de origem não traumática é ocorrência grave e não rara, que a cada ano acomete aproximadamente 30.000 americanos ${ }^{4}$. Apesar dos avanços nas técnicas diagnósticas, cirúrgicas e aportes perioperatórios, a mortalidade permanece em torno de $25 \%$ com morbidade ao redor de $50 \%$. Em estudos de epidemiologia, $25 \%$ dos pacientes morrem nas primeiras 24 horas após a HSA; $50 \%$ morrem no período de três meses e $64 \%$ de todas as mortes ocorrem na primeira semana". A HSA pode ser secundária a ruptura de um ou mais aneurismas ${ }^{1,2}$; pode ser espontânea (causa desconhecida), cuja incidência, na maioria dos estudos, é de $20 \%^{3,11}$, podendo ter associaçăo com fatores de risco como hipertensão arterial sistêmica, tabagismo e uso crônico do álcool ${ }^{4}$; a HSA também pode ser de origem traumática. Em 1924, Charles Symonds estabeleceu a retirada do líquido cefalorraquiano (LCR) através de punção lombar, como procedimento útil para 0 diagnóstico de HSA. Desde então, por muitos anos, o exame de LCR foi o melhor método para se confirmar diagnóstico de pacientes que apresentavam sinais e sintomas de HSA ${ }^{26,37}$. Muitos desses pacientes acabavam evoluindo com complicaçōes da punçāo lombar hoje bem conhecidas ${ }^{13}$,

Trabalho realizado no Setor de LCR da Disciplina de Neurologia da UNIFESP - Escola Paulista de Medicina: *Pós-Graduando da Disciplina de Neurologia; * Professor Adjunto da Disciplina de Neurologia e Chefe do Setor de LCR; ***Médico da Disciplina de Neurologia e Chefe do PS de Neurologia. Aceite: 5-março-1997. 
provavelmente devido ao fato de que as HSA podem ser acompanhadas de fatores agravantes tais como hipertensão intracraniana, hidrocefalia, hematomas intraparenquimatosos e ressangramento. Com o advento da angiografia cerebral em 1927, somou-se ao LCR outro exame capaz de diferenciar as HSA espontâneas daquelas causadas pela ruptura de aneurisma. Apesar de serem dois procedimentos invasivos, permaneceram em uso por muito tempo, até o advento da tomografia computadorizada (CT). A CT veio causar uma revolução no diagnóstico, na evolução e na abordagem dos pacientes com suspeitas de HSA ${ }^{33}$. A CT pode evidenciar imagem sugestiva de sangue nas cisternas basais, nos sulcos corticais, nos ventrículos, nos espaços subdurais, no parênquima cerebral, além de permitir correlação entre o local de sangramento e a possível localização de um ou mais aneurismas $^{10,23}$; ou de identificar também pela localização uma HSA de origem nāo aneurismática e de evolução benigna: a hemorragia perimesencefálica ${ }^{31}$. Deterioração neurológica, imediata ou tardia, pode resultar de hematomas, edema cerebral ou infartos por espasmos localizados ou generalizados. Nesses casos a CT veio mostrar grande papel como o exame mais sensível, com a vantagem de não ser invasivo, permitindo nāo só o diagnóstico da HSA, mas também de suas complicaçōes, evitando os efeitos indesejáveis da punção para a coleta do LCR.

Após a CT o papel da punção lombar como exame de primeira linha para o diagnóstico de HSA passou a ser questionado ${ }^{25}$, acreditando-se tratar de exame invasivo que poderia causar deterioração mais rápida do quadro neurológico, já tão comprometido dos pacientes com HSA, preferindo-se às vezes optar pela angiografia na vigência de quadro clínico característico ${ }^{18}$. Apesar da grande sensibilidade da CT, é sabido que há a possibilidade de ser exame inconclusivo ${ }^{1,2,23,25,30,37}$. Muitos casos que se apresentam com cefaléia aguda, associado a náuseas, vômitos, sinais meníngeos e alteração do nível de consciência , podem ser diagnosticados pela CT. Mas um grupo de pacientes (menos de 5\%) pode apresentar quadro clínico de HSA, sem imagem sugestiva de sangue na $\mathrm{CT}^{34}$. Nestes pacientes, o exame do LCR deve ser feito precocemente, respeitando-se o prazo de 12 horas depois do início dos sintomas, para se evitar falsa interpretação como acidente de punção, em virtude de não haver ainda xantocromia procedente de degradação de hemácias ${ }^{36,39}$.

O propósito deste trabalho é avaliar o valor do exame do LCR nos casos com suspeita de HSA, cuja CT de crânio não mostrou sinais de sangramento (CT negativa).

\section{MATERIAL E MÉTODOS}

Este estudo é baseado na análise de 23 casos de pacientes que apresentaram quadro clínico sugestivo de HSA e TC sem sinais de sangramento. Os pacientes foran submetidos a exame de TC de crânio em período de 24 hs a 15 dias após o início dos sintomas. Foi realizado exame do LCR através de punção lombar ou suboccipital no periodo de 24 hs a 15 dias após o ictus. Nenhum paciente apresentou contra indicação ao procedimento, que foi realizado logo após a avaliação da CT. A constatação de HSA pelo LCR foi baseada nas seguintes provas da veracidade da hemorragia: a) prova dos três tubos - o LCR apresenta uniformidade de turvação e coloração com contagem do número de hemácias semelhante nos três tubos; b) prova da sedimentação - não há formação de có́gulos ou retículo fibrinoso no LCR após a sedimentação das hemácias; c) prova da centrifugação - o líquido sobrenadante é limpido e xantocrômico após a separação das hemácias por centrifugação, imediatamente após a colheita do LCR. O grau de xantocromia foi avaliado segundo a escala de Bernheim.

Dos 23 pacientes, 20 realizaram angiografia cerebral para a constatação da presença ou não de aneurismas. Os aneurismas encontrados na angiografia cerebral foram classificados por territórios: a) carótida ( artéria comunicante posterior, artéria coroidéia, artéria oftálmica); b) cerebral anterior (anéria comunicante anterior); c) cerebral média; d) vértebro - basilar.

Os pacientes foram classificados clinicamente, antes da realização da CT, segundo os critérios estabelecidos por Hunt e Hess'". Ao exame clínico geral nenhum paciente se apresentou ictérico, e exames laboratoriais com dosagem de billirrubina mantiveram-se nos padrões normais.

\section{RESULTADOS}

Não ocorreu nenhum tipo de complicação associada à punçăo para colheita de LCR, seja lombar ou occipital. A distribuiçāo dos pacientes segundo sexo e idade mostrou predomínio do sexo 
Tabela 1. Distribuição dos pacientes em relação ao número de hemácias encontradas no $L C R$.

\begin{tabular}{cc}
\hline Hemácias $\left(\mathrm{mm}^{3}\right)$ & Pacientes $\left(\mathrm{N}^{\circ}\right)$ \\
\hline $0-1.000$ & 4 \\
$1.001-5.000$ & 6 \\
$5.001-10.000$ & 2 \\
$10.001-20.000$ & 6 \\
$20.001-40.000$ & 3 \\
$40.001-45.000$ & 2 \\
$>45.000$ & 0 \\
Total & 23 \\
\hline
\end{tabular}

Tabela 2. Resultado da angiografia cerebral realizada em 20 pacientes.

\begin{tabular}{ccc}
\hline & \multicolumn{2}{c}{ Pacientes } \\
& $\left(\mathrm{N}^{\circ}\right)$ & $(\%)$ \\
\hline Presença de aneurisma & 11 & 55 \\
Ausência de aneurisma & 7 & 35 \\
Vasoespasmo & 2 & 10 \\
Total & 20 & 100 \\
\hline
\end{tabular}

feminino na quinta e sexta década de vida. Um terço dos pacientes apresentou intervalo de tempo entre o ictus e a realização da CT e do

LCR entre 24 e 48hs, período de maior sensiblidade da CT e desses 50\% apresentaram diagnóstico de aneurisma na angiografia. Nāo houve correlação estatística entre o número de hemácias e: a presença de aneurisma, topografia dos aneurismas e classificação clínica dos pacientes. $\mathrm{O}$ número de hemácias encontradas não ultrapassou a 45000 hemácias $/ \mathrm{mm}^{3}$ (Tabela 1). $O$ índice de xantocromia de maior expressão no LCR foi 0,5. Dos 20 pacientes estudados com angiografia cerebral, 55\% tiveram o diagnóstico de aneurisma (Tabela 2) com predomínio no território de carótida e cerebral anterior (Tabela 3). O grau clínico dominante foi Hunt \& Hess I e II (Tabela 4). Houve predomínio na presença de aneurismas diagnosticados pela angiografia no grau clínico Hunt \& Hess I (Tabela 5).

\section{DISCUSSÃO}

A sensibilidade e eficácia da CT de crânio para o diagnóstico de HSA está muito bem definida na literatura 7,12,23,40. Alguns estudos mostram sensibilidade ao redor de $100 \%$ para visualização de sangue nas cisternas, quando o exame é realizado nas primeiras 24 horas de ictus ; outros apresentam sensibilidade de 90-95 \%2. Essa variação deve-se, provavelmente, ao tipo de aparelho utilizado, apesar de todos serem de terceira geraçāo. Mas sabe-se que, apesar da alta sensibilidade, um pequeno grupo de pacientes pode apresentar a combinação de sinais e sintomas sugestivos de HSA e apresentar a CT de crânio sem sinais de sangramento, o que não exclui o diagnóstico de HSA ${ }^{30,34}$. Nesses casos, o exame do LCR pode ser útil para a elucidaçăo do diagnóstico.

Tabela 3. Distribuição topográfica dos aneurismas.

\begin{tabular}{ll}
\hline Topografia & $\begin{array}{l}\text { Pacientes } \\
\left(\mathrm{N}^{\circ}\right)\end{array}$ \\
\hline Carótida & 5 \\
Cerebral anterior & 5 \\
Cerebral média & 0 \\
Vértebro-basilar & 3 \\
Total & $13^{*}$ \\
\hline
\end{tabular}

\footnotetext{
* Um paciente apresentou aneurismas múltiplos (total 3).
}

Tabela 4. Classificaçãos clínica dos pacientes segundo Hunt e Hess" no momento da realizaçāo da CT e do LCR.

\begin{tabular}{ccc}
\hline Grau clínico & $\begin{array}{c}\text { Pacientes } \\
\mathbf{N}^{\circ}\end{array}$ & $\begin{array}{c}\text { Pacientes } \\
\%\end{array}$ \\
\hline I & 12 & 52,2 \\
II & 7 & 30,4 \\
III & 4 & 17,4 \\
IV & 0 & 0 \\
V & 0 & 0 \\
Total & 23 & 100 \\
\hline
\end{tabular}


Tabela 5. Correlação entre o grau clínico (Hunt e Hess) no momento da realizaçāo da CT e do LCR com a presença ou näo de aneurisma(s).

\begin{tabular}{lcccccc}
\hline & Grau I & Grau II & Grau III & Grau IV & Grau V & Total \\
\hline Presença de aneurisma & 6 & 3 & 2 & 0 & 0 & 11 \\
Ausência de aneurisma & 3 & 4 & 0 & 0 & 0 & 7 \\
Total & & & & & $18^{*}$ \\
\hline
\end{tabular}

*Foram excluidos os pacientes que apresentaram vasoespamo na angiografia cerebral (Total de 2).

Os achados deste estudo servem para valorizar esse dado, salientando-se a escassez da literatura sobre o tema abordado. É importante frisar que, tanto quanto seja de nosso conhecimento, nenhum estudo na literatura analisou este aspecto nesse grupo de pacientes.

Os resultados obtidos nos casos estudados em relação ao sexo e faixa etária estão de acordo com os dados encontrados na literatura, nos quais há o predomínio do sexo feminino (2:1) e faixa etária com maior incidência na quinta e sexta décadas da vida, ${ }^{4,9,24}$. Foram agrupados os pacientes em relação ao intervalo de tempo entre o ictus e a realização do LCR e da CT, salientando que aproximadamente um terço dos pacientes apresentou intervalo de tempo entre 24-48 horas, ou seja, no período de maior sensibilidade da $\mathrm{CT}^{2,7,23}$. Mesmo assim, esta não foi conclusiva, tendo sido necessário o exame de LCR para firmar-se o diagnóstico de HSA. Mais de 50\% dos pacientes deste estudo tiveram o ictus entre 24 e 48 horas antes e apresentaram diagnóstico de aneurisma na angiografia cerebral. Os dois terços restantes, apresentaram intervalo de tempo que variou de 3-15 dias, confirmando a diminuiçāo de sensibilidade da CT após os primeiros 5 dias da instalação do quadro clínico e a importância da indicação do exame de LCR mesmo em fase mais tardia ${ }^{35}$. Sendo assim, se após a CT não fosse realizado o exame de LCR , esses pacientes provavelmente ficariam com outras hipóteses diagnósticas, não recebendo a abordagem adequada e cautelosa dos doentes que apresentam $\mathrm{HSA}^{4}$, ficando sujeitos a apresentar novo sangramento ou evoluir com complicações decorrentes da hemorragia ${ }^{24}$.

Existe discussão a nível de literatura sobre a possibilidade de ocorrer acidente de punção na coleta do LCR, falseando o diagnóstico de HSA ${ }^{6,25-27,30,36}$, submetendo o doente aos riscos e complicações da realização de uma angiografia cerebral desnecessária.

Os achados na literatura revelam que o ponto chave para a diferenciação é a presença de xantocromia; apenas Macdonald \& Mendelow2s discordam desta afirmação, valorizando a presença de hemácias. Tal trabalho foi criticado por Vermeulen et al. ${ }^{36}$ que reforçam o valor da xantocromia e revelam que apenas em periodo inferior a 12 horas do ictus o exame do LCR pode, mesmo na presença de HSA, não apresentar xantocromia ${ }^{3 y}$. Esse é o tempo em que transcorre a transformação de hemoglobina para oxi-hemoglobina e posteriormente bilirnubina ${ }^{5}$, através de enzima chamada hemeoxigenase (HO), produzida no espaço subaracnóide e no plexo coróide, demonstrado em modelo experimental em ratos . Em nosso estudo, a presença de xantocromia foi um dos critérios de inclusão, sendo o índice de 0,5 o de maior expressão, utilizando-se o método de visualização direta ${ }^{\mathbb{x}}$. Alguns autores contestam este método, acreditando ser pouco sensível e muito inferior à utilização da espectrofotometria ${ }^{6}$. No entanto, Page et al ${ }^{30}$ demonstraram que todos os indivíduos que apresentavam xantocromia visível tinham diagnóstico de hemorragia. A espectrofotometria tem grande utilidade nos casos sem a visualização de xantocromia ${ }^{n}$. Esses casos não foram incluídos neste estudo.

Mais recentemente têm-se utilizado outros métodos de análise do LCR para se firmar o diagnóstico de HSA, como a dosagem de ferritina e D-dimer, um anticorpo monoclonal altamente específico contra os produtos de degradação da fibrina humana ${ }^{15,16,20.38}$. Page et al. ${ }^{30}$ fizeram estudo 
comparativo, no exame de LCR para diagnóstico de HSA, entre a presença de xantocromia, dosagem de ferritina, utilização do D-dimer e presença de macrófagos fagocitando hemácias. Concluíram que a avaliação da xantocromia ainda é o método mais sensível para detectar a HSA.

Nas HSA causadas pela ruptura de aneurismas, 50\% dos pacientes admitidos em grandes centros neurológicos e neurocirúrgicos com diagnóstico de HSA apresentaram sintomas de aviso ("warning leak") em forma de episódios de pequenos sangramentos, dias ou mesmo semanas antes de ocorrer uma grande hemorragia". A cefaléia é o "sintoma de aviso" mais comum, ocorrendo em $90 \%$ dos $\operatorname{casos}^{14,21}$, sendo acompanhada em menor frequência por náuseas, vômitos, distúrbios visuais e sinais meníngeos. Muitas vezes esses sinais e sintomas são interpretados de forma errônea, sendo atribuidos a outras entidades como crise enxaquecosa, cefaléia tensional, sinusite aguda ou cervicalgia. O diagnóstico desses "sintomas de aviso"é de suma importância para que se possa alterar a história natural da HSA, diminuindo a mortalidade e a morbidade dos pacientes a partir do diagnóstico precoce, podendo a intervenção cirúrgica ser executada em condições satisfatórias para o paciente ${ }^{28}$.

Dados colbidos da literatura ${ }^{14,21,22,28,2 y}$ mostraram incidência maior dos "sintomas de aviso" em pacientes mais jovens, com discreto predomínio das mulheres com presença de aneurisma no território da artéria carótida. $O$ tempo decorrido entre estes pequenos sangramentos até a instalação de uma grande hemorragia variou de 7 a 28 dias, sendo, em média, 20 dias. Esses estudos ainda demonstraram que a sobrevida desses pacientes foi maior em relaçăo àqueles que não apresentaram " sintomas de aviso". Nesses casos, o exame do LCR é fundamental, pois a CT se apresentou normal (sem sinais de sangramento) em mais de $55 \%$ dos $\operatorname{casos}^{21}$, provavelmente devido à pequena quantidade de sangue encontrada; como também se demonstra em nosso estudo Tabela 4.

O número de hemácias não ultrapassou o valor de 45000 hemácias $/ \mathrm{mm}^{3}$ (Tabela 1), ficando a maioria dos pacientes na faixa de 1000 a 20000 hemácias $/ \mathrm{mm}^{3}$. Foi feita correlação entre o número de hemácias e: grau de comprometimento clínico; presença ou ausência de aneurisma e topografia dos aneurismas . Os achados não foram estatisticamente significantes, mas serviram para realçar o predomínio dos pacientes com Hunt \& Hess I e II na faixa de $0-20000$ hemácias $/ \mathrm{mm}^{3}$. Mais de $50 \%$ dos pacientes apresentaram os sinais e sintomas leves, todos classificados como grau I segundo Hunt e Hess, como mostra a Tabela 4; e destes $50 \%$ apresentaram aneurisma comprovado na angiografia cerebral (Tabela 5). Sem o exame de LCR estes pacientes ficariam sujeitos à evolução natural, piorando seu prognóstico.

A Tabela 2 demonstra que 11 pacientes (55\%) dos que realizaram angiografia cerebral apresentaram o diagnóstico de aneurisma. Nāo há dados significantes na literatura em relação à positividade de angiografia em pacientes com HSA, com CT de crânio sem sinais de sangramento. Em um estudo de Sadato et al. ${ }^{32}$, em um grupo de 20 pacientes, apenas 1 (5\%) apresentou aneurisma da junção vértebro-basilar, com CT sem sinais de sangramento. Outro estudo, realizado por van der Wee et al. ${ }^{34}$, revela a positividade de $100 \%$ de aneurisma por angiografia em dois pacientes que apresentaram CT de crânio sem sangramento, em um grupo de 119 pacientes com HSA. Em nosso estudo, um grupo de 7 pacientes (35\%) apresentou angiografia cerebral normal, podendo tratar-se de sangramento decorrente de hipertensão arterial sistêmica, ou mesmo de pequenas hemorragias perimesencefálicas, apesar de estas não terem sido diagnosticadas pela $C^{11,31}$. Apenas 2 pacientes (10\%) apresentaram vasoespasmos, os quais foram submetidos a novos exames a posteriore, cujos resultados não fazem parte deste estudo.

Em relação à topografia dos aneurismas a Tabela 3 mostra predomínio no território da artéria cerebral anterior e artéria carótida. Okawara"28, em estudo com 54 pacientes que apresentaram "warning leak" antes da HSA, observou predomínio dos aneurismas na topografia de carótida e artéria cerebral anterior. Estes fatos mostram indícios que nos aneurismas do território da artéria cerebral anterior e artéria carótida o "warning leak" é mais frequente. 
Em nosso estudo apenas 1 paciente apresentou mais de um aneurisma, o que é conflitante com os dados da literatura, que citam mais de um aneurisma em $30 \%$ dos casos ${ }^{19}$. $O$ nosso dado pode ser devido às condiçōes inerentes aos pacientes de nosso meio, ou ainda por nossa moderada casuística.

A maioria dos pacientes estudados apresentou sintomas leves, Hunt \& Hess Grau I ou II (Tabela 4), e apenas 4 apresentavam Hunt \& Hess Grau III ${ }^{17}$. Nenhum paciente apresentou Grau IV ou V, o que contraindicaria a punção suboccipital ou lombar para o exame de LCR, devido ao grande risco de complicações ${ }^{13.14}$. No entanto, o que deve ser ressaltado, devido a sua grande importância, é o fato de que os pacientes classificados como Hunt \& Hess I, em sua maioria absoluta, apresentaram aneurisma na angiografia cerebral (Tabela 5); esses pacientes, em circunstâncias outras, poderiam ter recebido alta hospitalar após realizaçāo da CT (sem o exame de LCR) com outros diagnósticos, podendo retornar futuramente com um quadro clínico mais grave, tolhendo-lhes assim a possibilidade de intervenção cirúrgica em condições satisfatórias.

Sendo assim, o exame de LCR deve ser indicado para todo paciente que apresentar sinais e sintomas sugestivos de HSA e que apresentar CT de crânio sem sinais de sangramento; os casos que apresentarem contra indicação para o procedimento, devem aguardar a melhora dos sintomas, antes de se proceder à punção. A realização de exame de LCR, além de poder dar com certeza o diagnóstico de HSA, faz com que pacientes, com exame de LCR negativo para HSA, não sejam submetidos a angiografia cerebral, afastando-os dos riscos desse procedimento invasivo.

\section{REFERÊNCIAS}

1. Adams HP, Jergenson DD, Kassel NF, Sahs AL. Pitfalls in the recognition of subarachnoid haemorrhage. JAMA 1980;244:794-796

2. Adams HP, Kassell NF, Torner JC, Sahs AL. CT and clinical correlations in recent aneurysmal subarachnoid hemorrhage: a preliminary report of the Cooperative Aneurysm Study. Neurology 1983;33:981-988.

3. Alexander MSM, Dias PS, Uttley D. Spontaneous subarachnoid haemorrhage and negative cerebral panangiography: review of 140 cases. J Neurosurg 1986;64:537-542.

4. American Heart Association Stroke Council. Guidelines for the management of aneurysmal subarachnoid hemornhage. Stroke 1994;25:2314-2324.

5. Barrows LJ, Hunter FT, Banker BQ. The nature and clinical significance of pigments in the cerebrospinal fluid. Brain 1955;78:59-80.

6. Beetham R. Spectrophotometric examination of CSF for xanthochomia. Lancet 1992;339:1492.

7. Bell BA, Kendall BE, Symon L. Computed tomography in aneurysmal subarachnoid haemorrhage. J Neurol Neurosurg Psychiatry1 980;43:522-524.

8. Bernhein AR. The icterus index. JAMA 1924;82:291.

9. Bonita R, Thomsom S. Subarachnoid haemonthage: epidemiology, diagnosis, management and outcome. Stroke 1985;16:591-594.

10. Borri ML. A tomografia computadorizada do crânio na hemorragia subaracnoídea por aneurisma: análise de 100 casos. Tese de Mestrado, UNIFESP - EPM. Sāo Paulo, 1990.

11. Cioffi F, Pasqualine A, Cacazzani P, Dapian R. Subarachnoid haemorrhage of unknown origin: clinical and tomographical aspects. Acta Neurochir. (Wien) 1989;97:31-39.

12. Davis KR, New PFJ, Ojemann RG, Crowell RM, Morawetz RB, Roberson GH. Computed tomographic evaluation of haemorrhage secondary to intracranial aneurysm. Am J Roentgenol 1976;127:143-153.

13. Duffy G. P.Lumbar puncture in spontaneous subarachnoid haemorrhage. Br Med J 1982;285:1163-1164.

14. Duffy GP. The"Warning leak"in spontaneous subarachnoid haemorrhage. Med J Aust 1983;1:514-516.

15. Eclache V, Vu T, Le Roux G. D-dimers levels in the cerebrospinal fluid: a marker of central nervous system involvement in neoplastic disease. Nouv Rev Fr Hematol 1994;36:321-324.

16. Greenberg CS, Devine DV, McCrae KM. Measurement of plasma fibrin D- dimer levels with the use of a monoclonal antibody coupled to latex beads. A J C P 1987;87:94-99.

17. Hunt WE, Hess RM. Surgical risk as related to time of intervention in the repair of intracranial aneurysm. J Neurosurg 1968:28:14-20.

18. Ivan LP. Computed tomographys and the early diagnostic lumbar puncture. Can Med Assoc J 1979;121:1442.

19. Kendall $\mathrm{BE}$, Lee $\mathrm{BCP}$, Claveria $\mathrm{E}$. Computerized tomography and angiography in subarachnoid haemorrhage. $\mathrm{Br} \mathrm{J}$ Radiol 1976;49:483-501.

20. Lang DT, Berberian LB, Stephein L, Ault M. Rapid differentiation of subarachnoid hemorrhage from traumatic lumbar puncture using the D-dimer assay. A J C P 1990;93: 403-405.

21. Leblanc $R$. The minor leak preceding subarachnoid hemorrbage. J.Neurosurg.1987;66:35-39.

22. Leblanc $\mathbf{R}$, Winfield DJA. The warning leak in subarachnoid hemorrhage and the importance of its early diagnosis. Can Med Assoc J 1984;131:1235-1236.

23. Liliequist B, Lindquist M,Valdimarsson E. Computed tomography and subarachnoid hemornhage. Neuroradioloy 1977;14:21-26. 
24. Locksley HB. Natural history of subarachnoid hemornage, intracranial aneurysms and arteriovenous malformations. $J$ Neurosurg 1966;24:779-780.

25. MacDonald A, Mendelow AD. Xanthochromia revisited: a re-evaluation of lumbar puncture and $\mathrm{CT}$ scanning in the diagnosis of subarachnoid haemomhage. J Neurol Neurosurg Psychiatry 1988;51:342-344.

26. Manton KI, Gean AD. The spinal tap: a new look at an old test. Ann Intern Med 1986; 104:840-848.

27. Mendelow AD, Cartidge N. Xanthochromia in subarachnoid haemonthage. J Neurol Neurosurg Psychialry 1990;53:270-271.

28. Oknwara SH. Warning signs prior to rupture of an intracranial aneurysm. $J$ Neurosurg 1973;38:575-580.

29. Ostergaard JR. Headache as a warning symptom of impending aneurysmal subarachnoid haemorrhage. Cephalalgia 1991;11:53-55.

30. Page KB, Howell SJ, Smith CML, Dabbs DJW, Malia RG, Porter NR, Thickett KJ, Wilkinson GM. Bilirubin, ferritin, D. dimers and erythrophages in the cerebrospinal fluid of patients with suspected subarachnoid haemorrhage but negative computed tomography scans. J Clin Pathol 1994:47:986-989.

31. Rinkel GJE, Wijdicks EFM, Vermeulen M, Ramos LMP, Tanghe HLJ, Hasan D, Meiners LC, Van Gijn J. Nonaneurysmal perimesencephalic subarachnoid hemonhage : CT and MR patterns that differ from aneurysmal nupture. A J N R 1991;12:829-834.

32. Sadato N, Numaguchi Y, Riganonti D, Salcman M, Gellad F.E, Kishikawa T. Bleeding patterns in ruptured posterior fossa aneurysms: a CT study. J Comput Assist Tomogr 1991;15:612-617.

33. Scote WR, New PFJ, Davis KR. Computerized axial tomography of intracerebral and intraventricular hemorrhage. Radiology 1974;122:73-80.

34. Van Der Wee N, Rinkel GJE, Hasan D, Van Gijn J. Detection of subarachnoid haemorrhage on early CT: is Jumbar puncture still needed after a negative scan? J Neurol Neurosurg Psychiatry 1995;58:357-359.

35. Van Gijn J, Van Dongen KJ. The time course of aneurysmal haemorrhage on computed tomograns. Neuroradiology $1982 ; 23: 153-156$.

36. Vermeulen M, Hasan D, Blijenberg BG, Hijdra A, Van Gijn J. Xanthochromia after subarachnoid haemorthage needs no revisitation. J Neurol Neurosurg Psychiatry 1989;52:826-828.

37. Vermeulen M, Van Gijn J. The diagnosis of subarachnoid haemonthage. J. Neurol. Neurosurg. Psychintry 1990;53:365-372.

38. Vermeulen M, Vavliet HHDM, Lindsay KW, Hijdra A, Van Gijn J. Source of fibrin / fibrinogen degradation products in the CSF after subarachnoid hemorrhage. J Neurosurg 1985;63:573-577.

39. Walton JN. Subarachnoid haemorrhage. Edinburgh: Livingstone, 1956.

40. Weisberg LA. Computed tomography in aneurysmal subarachnoid hemorrhage. Neurology 1979;29:802-808. 